\title{
Molecular epidemiology of non-viral sexually transmitted infections in the central Alpine province of Bolzano, northern Italy from April 2016 to March 2017
}

\author{
Richard Aschbacher, ${ }^{1}$ Francesca Romagnoli, ${ }^{1}$ Elisa Masi, ${ }^{1}$ Valentina Pasquetto, ${ }^{1}$ Franco Perino, ${ }^{2}$ \\ Klaus Eisendle, ${ }^{2,3}$ Monica Braghetto, ${ }^{4}$ Sergio Messini, ${ }^{4}$ Serena Delbue, ${ }^{5}$ Elisabetta Pagani ${ }^{1}$ \\ ${ }^{1}$ Laboratory of Microbiology and Virology, Bolzano Central Hospital; ${ }^{2}$ Department of Dermatology, Venereology and \\ Allergology, Academic Teaching Department of Medical University Innsbruck, Bolzano Central Hospital; ${ }^{3}$ College of \\ Health Care Professions Claudiana, Bolzano; ${ }^{4}$ Department of Gynecology and Obstetrics, Bolzano Central Hospital; \\ ${ }^{5}$ Department of Biomedical, Surgical and Dental Sciences, University of Milan, Italy
}

\section{Summary}

Chlamydia trachomatis, Neisseria gonorrhoeae, Trichomonas vaginalis, Ureaplasma parvum, Ureaplasma urealyticum, Mycoplasma hominis and Mycoplasma genitalium are established or presumed STI pathogens. The present study aims to describe the one-year molecular epidemiology of these seven pathogens in the Province of Bolzano, Northern Italy. From April 2016 to March 2017, 2,949 patients, mainly females, were enrolled and 3,427 urine, vaginal, endocervical and/or urethral samples were subjected to simultaneous analysis of the seven pathogens by means of Real Time Polymerase Chain Reaction (Anyplex ${ }^{\mathrm{TM}}$ II STI-7 Detection Kit Seegene, Seoul, Korea). At least one of the

Correspondence: Elisabetta Pagani, Laboratory of Microbiology and Virology of the Bolzano Central Hospital, via Amba Alagi 5, 39100 Bolzano (BZ), Italy.

Tel.: +39.471907324 - Fax +39471272631

E-mail: elisabetta.pagani@sabes.it

Key words: STI, Multiplex RT-PCR, Ureaplasma, Mycoplasma, Chlamydia, Gonococcus, Trichomonas.

Acknowledgments: the authors wish to thank the staff of the units who participate in this study.

Contributions: the authors contributed equally.

Conflict of interest: the authors declare no potential conflict of interest.

Funding: none.

Received for publication: 27 September 2018.

Revision received: 10 October 2018.

Accepted for publication: 10 October 2018.

(C) Copyright R. Aschbacher et al., 2018

Licensee PAGEPress, Italy

Microbiologia Medica 2018; 33:7852

doi:10.4081/mm.2018.7852

This article is distributed under the terms of the Creative Commons Attribution Noncommercial License (by-nc 4.0) which permits any noncommercial use, distribution, and reproduction in any medium, provided the original author(s) and source are credited. seven microorganisms were detected in $40.7 \%$ of patients, with uneven distribution: $43.1 \%$ in females $(\mathrm{F})$ and $29.8 \%(\mathrm{P}<0.001)$ in males (M). The prevalence of microorganisms was as follows: $30.3 \%$ U. parvum (F: 35.6\%, M: 8.3\%), 6.9\% U. urealyticum (F: 6.8\%, M: 7.0\%), 4.9\% M. hominis (F: 5.4\%, M: 2.3\%), 4.9\% C. trachomatis (F: 3.4\%, M: 11.4\%), 1.1\% M. genitalium (F: 1.0\%, M: $1.2 \%$ ), $1.2 \%$ N. gonorrhoeae (F: $0.17 \%, \mathrm{M}: 5.6 \%$ ) and $0.40 \%$ T. vaginalis (F: $0.38 \%$, M: $0.53 \%$ ). Mixed infections were detected in $7.4 \%$ of patients. Highest prevalence was observed for $U$. parvum, followed by $U$. urealyticum and $M$. hominis and significant presence of multi-pathogen infections was registered.

\section{Introduction}

Viral agents are the predominant cause of sexually transmitted infections (STIs) worldwide, with more than 500 million people living with genital Herpes simplex (HSV) infection and more than 290 million women with a human Papillomavirus (HPV) infection (http://who.int/reproductivehealth/topics/rtis/en/). Nevertheless, non-viral STIs of the female and male urogenital tract are also major causes of morbidity among sexually active individuals all over the world and are generally caused by various bacterial and protozoal agents. T. vaginalis is a very common protozoal agent of STIs (8), C. trachomatis is the most frequently reported STI-microorganism in Europe $(1,9,11,13)$, and $N$. gonorrhoeae is the well-defined etiological agent of gonorrhea (6). Most recent epidemiological data report the occurrence of an estimated 357 million new infections worldwide with one of the four following STIs: chlamydia (131 million), gonorrhoea (78 million), syphilis (5.6 million) and trichomoniasis (143 million) (http://who.int/reproductivehealth/topics/rtis/en/).

Among other bacterial agents, Ureaplasma parvum, Ureaplasma urealyticum, Mycoplasma hominis and Mycoplasma genitalium are associated with the development of STIs. They inhabit the genitourinary tract, and infections by these agents are relevant mainly in parturient women and their fetuses and newborns. Nevertheless, their precise role in many STIs remains unproven, due to the fact that they are part of the normal genital microbiota of sexually active women and men and a specific relationship between their presence and the disease is often difficult to prove $(3,7,10,14,15,17)$ New molecular tools have been developed during the past few years in order to improve the laboratory diagno- 
sis of STIs, with the specific aim of allowing the simultaneous detection of more than one pathogenic agent in clinical specimens (16). This allows to save time for diagnosis and to better study the epidemiological profile of STIs in a specific geographic area.

The present retrospective study describes the one-year epidemiology of seven bacterial and protozoal species (U. parvum, $U$. urealyticum, $M$. hominis, $M$. genitalium, C. trachomatis, $N$. gonorrhoeae and $T$. vaginalis), recovered from urine, vaginal, endocervical and/or urethral samples collected from patients in the local province of Bolzano. Bolzano province is an Alpine mountain region, located in northern Italy, near the Austrian border (population of 525,000 inhabitants at 2016 census). The laboratory of Microbiology and Virology, Bolzano Central Hospital, is the only referral center in the whole Bolzano province and collects all specimens from different regional hospitals and local practitioners.

\section{Materials and Methods}

\section{Study population}

Specimens from all 2,949 patients (80.8\% females), referring to the Laboratory of Microbiology and Virology, Bolzano Central Hospital, were enrolled in this study from April 2016 to March 2017. Age distribution of the patients was as follows: $0-10$ years (F: $0.3 \%, \mathrm{M}: 2.6 \%$ ), 11-20 years (F: $6.2 \%, \mathrm{M}: 4.0 \%), 21-30$ years (F: 36.5\%, M: 21.4\%), 31-40 years (F: 41.6\%, 23.0\%), 41-50 years (F: $10.3 \%, 16.0 \%), 51-60$ years $(\mathrm{F}: 1.9 \%, \mathrm{M}: 9.4 \%),>60$ years $(\mathrm{F}$ : $1.2 \%, \mathrm{M}: 6.9 \%$ ); for $1.8 \%$ of females and $16.5 \%$ of males the age was not specified.

Demographic and clinical data of the patients are summarized in Table 1. Specifically, 429 patients were referred from the Dermatology Clinic, 388 from the Gynecology/Obstetric Clinic, 43 from other hospital clinics and 2,089 were outpatients. A total of 3,427 clinical specimens, comprising 710 first-voided urine samples, 308 vaginal swabs, 2,150 cervical swabs, 191 urethral swabs, and 68 other samples were collected, refrigerated and transferred within 4 hours to the Hospital Laboratory of Microbiology and Virology.

\section{DNA isolation from clinical specimens}

First-voided urine samples were collected in a sterile screwcap plastic disposable container (FL medical Torreglia, Italy), whereas vaginal, endocervical and urethral specimens were collected using the swabs provided by the commercial kit eNAT ${ }^{\mathrm{TM}} 2$
(Copan Diagnostics, Brescia, Italy). After collection, samples were processed within 72 hours.

DNA isolation was performed using the STARMag Tissue Cartridge Kit (Seegene, Seoul, Korea) on the Microlab ${ }^{\circledR}$ NIMBUS IVD system (Hamilton Diagnostics, USA), following manufacturer's instructions.

\section{Detection of bacterial and protozoan genomic sequences}

Simultaneous analysis for detection of genomic sequences belonging to several different non-viral STIs agents (U. parvum, U. urealyticum, M. hominis, M.genitalium, C. trachomatis, $N$. gonorrhoeae, T.vaginalis) was conducted by means of the multiplex real-time PCR assay Anyplex ${ }^{\mathrm{TM}}$ II STI-7 Detection Kit V1.1 (Seegene, Seoul, Korea), whose method is based on the tagging oligonucleotide cleavage and extension technology (TOCE), and run on the CFX96 real-time thermocycler (BioRad Hercules, CA, USA), in accordance with manufacturer's protocols. Positivity was defined as test results,+++++ or + , according to the manufacture's manual. The significance of proportion differences was analyzed with the proportion comparison test, using the Medcalc $₫$ software version 15.11.4 (MedCalc software, Ostend, Belgium).

\section{Results}

The prevalence of studied STI pathogens is summarized in Table 2. Overall, at least one of the seven STI microorganisms was detected in $40.7 \%$ of patients, with a strong uneven distribution: $43.1 \%$ among females and $29.8 \%$ among males $(\mathrm{P}<0.001)$, whereas mixed infections, caused by different combinations of pathogens, were detected in $7.7 \%$ of females vs. $6.5 \%$ of males $(\mathrm{P}=0.33)$. Combinations of mixed infections are reported in Figure 1.

$U$. parvum was detected in $30.3 \%$ of patients $(35.6 \%$ of females vs. $8.3 \%$ of males; $\mathrm{P}=0.001$ ) and it had the highest prevalence among all of the analyzed pathogens, followed by $U$. urealyticum, detected in $6.9 \%$ of patients $(6.8 \%$ of females $v s .7 .0 \%$ of males; $\mathrm{P}=0.88$ ), and by $C$. trachomatis, detected in $4.9 \%$ of patients (3.4\% of females vs. $11.4 \%$ of males; $\mathrm{P}<0.001)$. Similarly, M. hominis was found in $4.9 \%$ of patients $(5.4 \%$ of females vs. $2.3 \%$ of males; $\mathrm{P}=0.001)$. The other microorganisms were detected in about $1 \%$ or less of patients (Table 2 ).

Prevalence of STI pathogens for females peaked in the age group 21-30 years for U. urealyticum, M. hominis, M. genitalium and $C$. trachomatis; for males a similar age distribution was found

Table 1. Patient demographic and clinical specimen data.

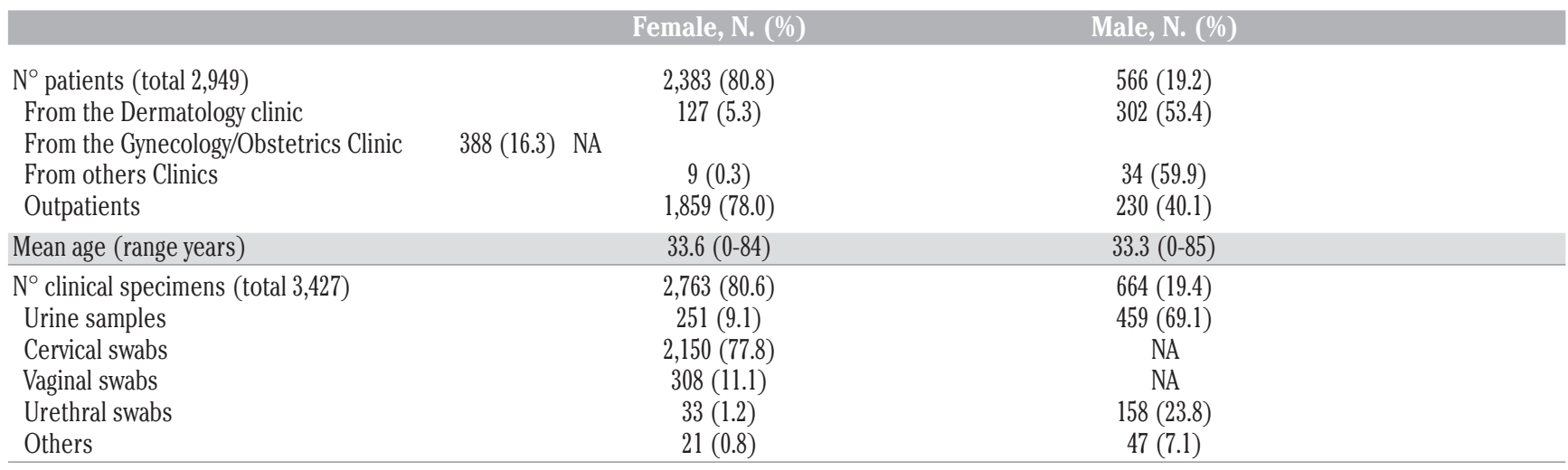

NA: not applicable. 
for $U$. urealyticum, U. parvum and C. trachomatis, whereas for $N$. gonorrhoeae the peak prevalence was shifted to the age group 3140 years. No clear age group distribution was detectable for the few $T$. vaginalis infections in both sexes and for $N$. gonorrhoeae in females, because of the very low number of positive patients (data not shown).

\section{Discussion}

Molecular methods are the new "gold standard" for laboratory testing of many STIs. Our study showed the detection of at least one of the seven studied non-viral STI microorganisms in $40.7 \%$ of patients referring to the Hospital of Bolzano, Northern Italy. These findings are similar to those from recent epidemiological surveys that have been conducted in Italy, employing molecular methods for multiplex detection of pathogens. Avolio et al., among a population of more than 2,700 patients from a different area of Northern Italy, observed a prevalence of $48.2 \%$ for at least one of the seven microorganisms, which are the targets of the Anyplex ${ }^{\mathrm{TM}}$ II STI-7 assay (2). Among a smaller population from Southern Italy (1,580 patients), Del Prete et al. reported a lower prevalence of the seven targeted microorganisms with $30.7 \%$ among the enrolled females and $21.1 \%$ among the enrolled males (4).

We observed a higher prevalence of $U$. parvum and $U$. urealyticum in our specimen collection, followed by M. hominis, compared to the other studied species. Additionally, the distribution of $U$. parvum was significantly different between females and males. These findings confirmed two very recent Italian surveys $(2,4)$.

Both, ureaplasma and mycoplasma species, are suspected to play a role in the development of several pathological conditions of the genital tract of adults and in newborn infections; nevertheless, because of their concomitant commensal role in the genital district, it is hard to discriminate pathogenic from commensal behavior $(3,7,10,14,15,17)$. The association between genital mycoplasmas and ureaplasmas and the risk of male infertility, relevant for the possible treatment of patients with infertility, has been studied widely (7).

C. trachomatis is the major known etiological agent of STIs;

Table 2. Prevalence of seven non-viral STI microorganisms in clinical specimens collected from female and male patients referring to the Laboratory of Microbiology and Virology of the Bolzano Hospital, Northern Italy.

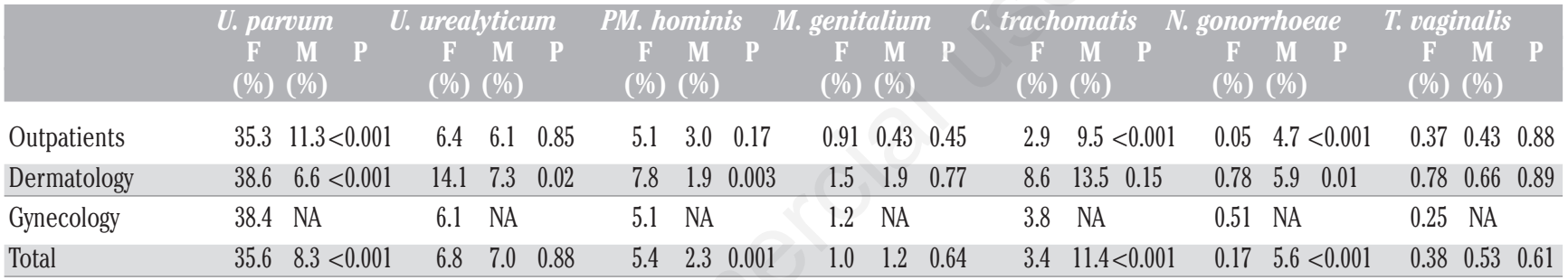

NA: not applicable.

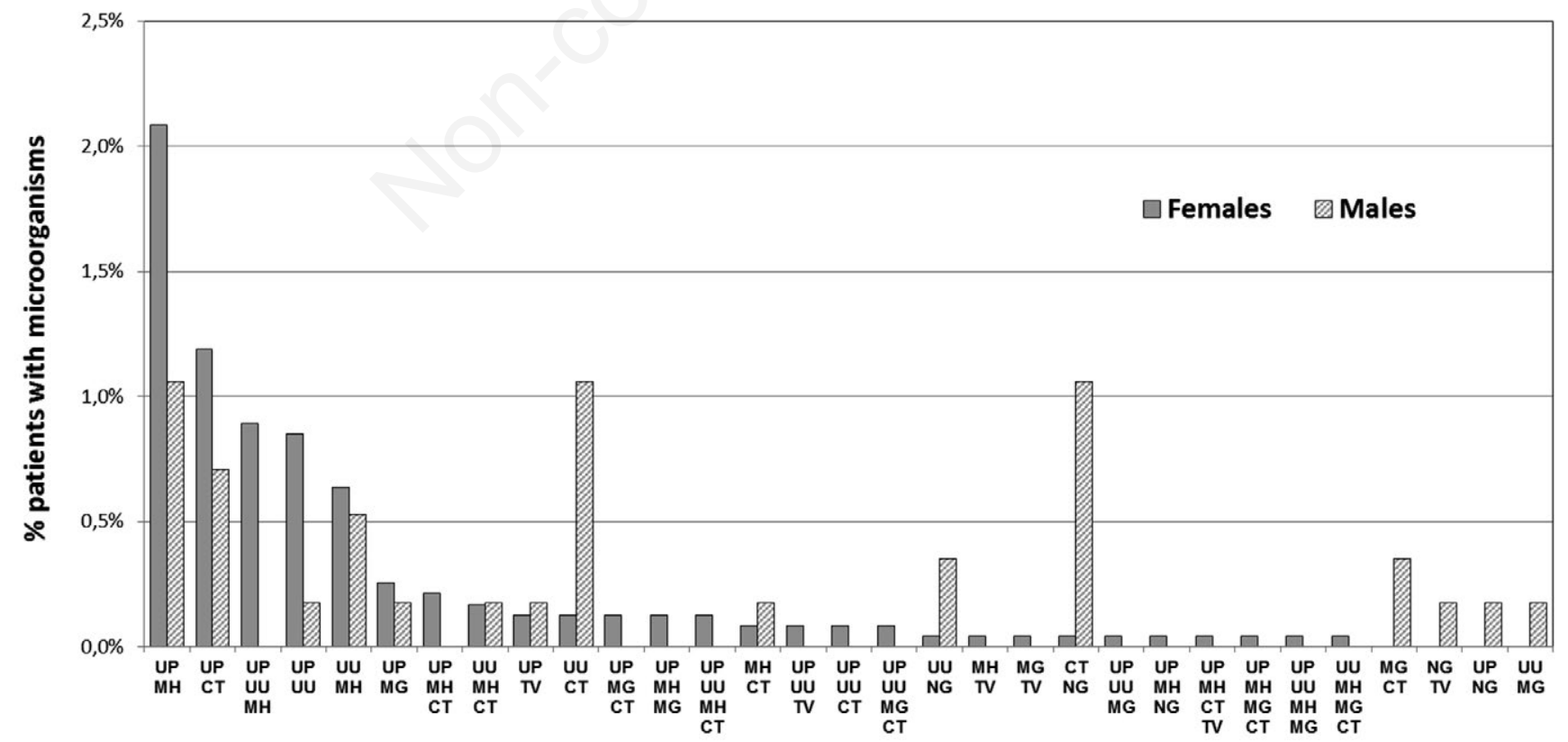

UP: U. parvum, UU: U. urealyticum, MH: M. hominis, MG: M. genitalium, CT: C. trachomatis, NG: N. gonorrhoeae, TV: T. vaginalis

Figure 1. Prevalence of mixed infections caused by STI microorganisms. 
its prevalence is highly variable among both sexually active females and males, ranging from $0.2 \%$ to $8.0 \%$, as reported by various European studies (13). With regard to the Italian situation, the two already cited studies reported a prevalence of $1.8 \%-2.6 \%$ among females and of $3.6 \%$ among males $(2,4)$. Another survey, referring to a high-density urban area in Northern Italy, and employing a different PCR method, reported a much higher C. trachomatis prevalence of $8.1 \%$, with a male/female ratio of 1.08 , and with higher distribution within patients referring to the STI department compared to patients referring to the gynecology department (5). Similarly, we found a significantly higher prevalence of $C$. trachomatis in male (11.4\%) compared to female $(3.4 \%)$ patients, presenting either as single infection or in association with $U$. urealyticum or $N$. gonorrhoeae. Nevertheless, it should be underlined that the observed relative frequencies may be biased, since male patients were mainly outpatients or referred from the Dermatology department, for a possible diagnosis of STIs, whereas female patients were mainly subjected to the C. trachomatis test as part of routine pregnancy screening.

$N$. gonorrhoeae infection represents one of the most severe health issues worldwide, especially in light of its increasing resistance to currently used antibiotics: according to the 2005-2012 World Health Organization (WHO) report, global prevalence of $N$. gonorrhoeae was $0.8 \%(0.3 \%$ to $1.7 \%)$ among women and $0.6 \%(0.3 \%$ to $1.0 \%$ ) among men (12). An Italian study found a much higher infection rate among men (3.6\%) than among women (0.16\%) (4). Similarly, we found a significantly higher prevalence in males (5.6\%) compared to females $(0.17 \%)$. Interestingly, another Italian study, conducted on 2,735 endocervical specimens collected from childbearing age women from Northern Italy, and performed using the same Multiplex Real Time PCR assay as used in our study, reported a complete absence of $N$. gonorrhoeae infection (2).

Peaking prevalence of genital mycoplasms and C. trachomatis in the age groups 21-30 years for males and females probably reflects the frequency of sexual activity in both sexes and the pregnancy screening in females in this age group; shifting of the peak for $N$. gonorrhoeae infections in males to the age group 31-40 years probably reflects the increasing risk factors for STIs in the local male population, depending on their possible sexual habits.

The global prevalence of $T$. vaginalis infections has been estimated around 5.0\% (1.0-11.5\%) in women and 0.6\% (0.1-1.3\%) in men (12). A study referring to a population from Southern Italy observed prevalence values of $1.5 \%$ among women and $1.3 \%$ among males (4), whereas a study referring to a population from Northern Italy found a prevalence of $0.9 \%$ in women (2). In our study, a lower prevalence was observed.

This study has the following limitations: i) This is a retrospective study; ii) The analysis focused on only seven non-viral pathogens; iii) Clinical data from the enrolled subjects were not collected, and any possible risk factors or clinical signs and symptoms could not be taken into account; iv) The results obtained by the newly introduced Multiplex Real Time PCR were not compared with those obtained with other methods.

\section{Conclusions}

On the whole, epidemiological data regarding the distribution of seven non-viral STI related pathogens in the Province of Bolzano, Northern Italy, was described for the first time: Ureaplasma species were prevalent, but presence of co-infections with $U$. parvum, U. urealyticum and M. hominis were frequent. Real Time PCR Multiplex assay enables a fast and simultaneous identification of different etiological agents of STIs, reducing the risk of sequelae and complications for the patients.

\section{References}

1. Amato-Gauci AJ, Spiteri G. Surveillance report: Sexually transmitted infections in Europe 2013. Available from: http://www.ecdc.europe.eu

2. Avolio M, Modolo ML, Stano P, et al. Molecular Evaluation of 7 sexually transmissible microorganisms in symptomatic and asymptomatic Italian childbearing age women: Is Ureaplasma parvum a real innocent bystander? Microbiol Med 2016;31:3.

3. Capoccia R, Greub G, Baud D. Ureaplasma urealyticum, Mycoplasma hominis and adverse pregnancy outcomes. Curr Opin Infect Dis 2013;26:231-40.

4. Del Prete R, Ronga L, Lestingi M, et al. Simultaneous detection and identification of STI pathogens by multiplex RealTime PCR in genital tract specimens in a selected area of Apulia, a region of Southern Italy. Infection 2017.

5. Foschi C, Nardini P, Banzola N, et al. Chlamydia trachomatis infection prevalence and serovar distribution in a high-density urban area in the north of Italy. J Med Microbiol 2016;65:510-20.

6. Hill SA, Masters TL, Wachter J. Gonorrhea - an evolving disease of the new millennium. Microb Cell 2016;3:371-89.

7. Huang C, Zhu HL, Xu KR, et al. Mycoplasma and ureaplasma infection and male infertility: a systematic review and metaanalysis. Andrology 2015;3:809-16.

8. Kissinger P. Trichomonas vaginalis: a review of epidemiologic, clinical and treatment issues. BMC Infect Dis 2015;15:307.

9. Mackern-Oberti JP, Motrich RD, Breser ML, et al. Chlamydia trachomatis infection of the male genital tract: an update.J Reprod Immunol 2013;100:37-53.

10. Murtha AP, Edwards JM. The role of Mycoplasma and Ureaplasma in adverse pregnancy outcomes. Obstet Gynecol Clin North Am 2014;41:615-27.

11. Mylonas I. Female genital Chlamydia trachomatis infection: where are we heading? Arch Gynecol Obstet 2012;285:1271-85.

12. Newman L, Rowley J, Vander Hoorn S, et al. Global estimates of the prevalence and incidence of four curable sexually transmitted infections in 2012 based on systematic review and global reporting. PLoS One 2015;10:e0143304.

13. Redmond SM, Alexander-Kisslig K, Woodhall SC, et al. Genital chlamydia prevalence in Europe and non-European high income countries: systematic review and meta-analysis. PLoS One 2015;10:e0115753.

14. Sweeney EL, Dando SJ, Kallapur SG, Knox CL. The Human ureaplasma species as causative agents of chorioamnionitis. Clin Microbiol Rev 2016;30:349-79.

15. Taylor-Robinson D, Jensen JS. Mycoplasma genitalium: from Chrysalis to multicolored butterfly. Clin Microbiol Rev 2011;24:498-514.

16. Unemo M, Ballard R, Ison C, et al. Laboratory diagnosis of sexually transmitted infections, including human immunodeficiency virus. World Health Organization 2013. Available from: http://apps.who.int/iris/bitstream/10665/85343/1/9789241505 840 _eng.pdf

17. Viscardi RM. Ureaplasma species: role in neonatal morbidities and outcomes. Arch Dis Child Fetal Neonatal Ed 2014;99:F87-92.

18. World Health Organization. Sexually transmitted infections report. 2018. http://who.int/reproductivehealth/topics/rtis/en/ 